\title{
La innovación docente en Hidrología. Aplicación práctica
}

\author{
ISABel CLARA Rodríguez Medina \\ Universidad de Sevilla \\ Departamento de Ingeniería \\ Aeroespacial y Mecánica de Fluidos \\ irodriguez8@us.es \\ D.O.I.: http://dx.doi.org/10.12795/JDU.2018.i01.37 \\ Pp.: 655-670
}

\section{Resumen}

Se presenta la aplicación de un Ciclo de Mejora Docente desarrollado durante el curso 2017-2018 en "Hidrología”, asignatura del Grado de Ingeniería Civil. El modelo metodológico empleado es el de reelaboración de ideas. Se presenta un mapa de contenidos diseñado y una relación detallada de actividades. Como elemento innovador principal se introduce la realización de varios "talleres conceptuales" de manera "on-time" y "on-line", en los que se hace el estudio de una cuenca hidrológica. Para evaluar la evolución del conocimiento de los alumnos se realizan cuestionarios inicial y final y se hace una comparación entre ambos. Como conclusiones es destacable que la aplicación de los "talleres conceptuales" o dar "clase con la boca cerrada" permiten realizar una docencia más didáctica y satisfactoria para los alumnos en los contenidos aprendidos y en la forma en que se han aprendido, como queda reflejado en los cuestionarios.

Palabras clave: Hidrología, Grado en Ingeniería Civil, Docencia Universitaria, Innovación Docente, Cuenca Hidrológica. 


\section{Breve descripción del contexto y modelo metodológico}

El ciclo de mejora se ha realizado en la asignatura de Hidrología, enseñanza que se imparte en la Escuela Técnica Superior de Ingenieros de la Universidad de Sevilla dentro del Grado de Ingeniería Civil. La asignatura está organizada por bloques temáticos, todos ellos con un carácter teórico-práctico.

El segundo bloque temático de la asignatura, "La Cuenca Hidrológica", es el elegido para la aplicación de este ciclo de mejora. El desarrollo del ciclo completo ha tenido una duración de 2 semanas y al tener asignada la asignatura 4 horas semanales se han empleado 4 sesiones de 2 horas cada una. La primera sesión se imparte en un aula normal y las restantes, segunda, tercera y cuarta, se imparten en el Centro de Cálculo, en el que se desarrollan los llamados "talleres conceptuales". Al tener este bloque temático un carácter eminentemente práctico, se ha diseñado de tal forma que cada alumno utiliza un ordenador con software específico, con el que se realizan diversas actividades en varias sesiones y en la evaluación final. El esquema del bloque temático se recoge en la Figura 1.

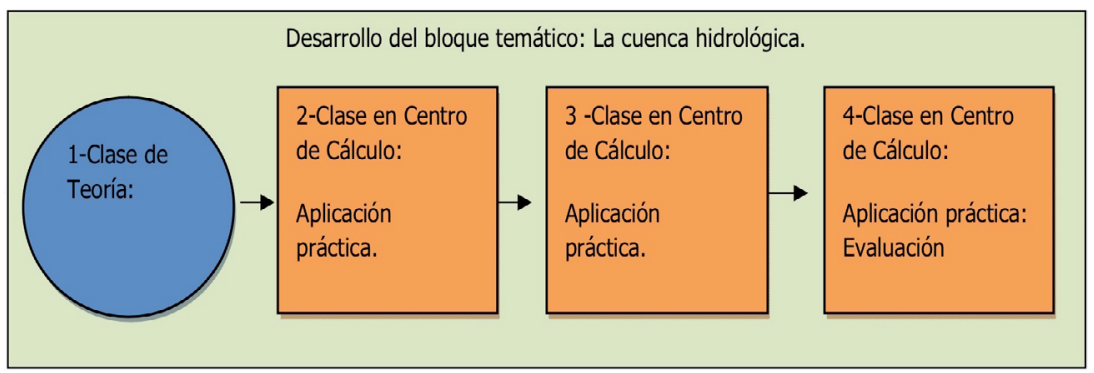

Figura 1. Esquema general del bloque temático "La Cuenca Hidrológica".

El modelo metodológico posible diseñado es el basado en la reelaboración de ideas de los alumnos a partir del planteamiento de un problema (De Alba y Porlán, 2017), es decir, se abre la clase con el planteamiento de un problema seguido de la exploración de ideas de los alumnos, 
después se realizan actividades de contraste y por último se extraen las conclusiones. A continuación se representa un esquema con el modelo metodológico utilizado (Figura 2).

Modelo metodológico aplicado:

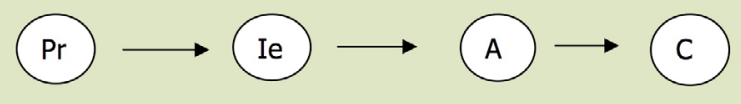

Figura 2. Modelo metodológico empleado.

\section{Diseño del Ciclo de Mejora Docente}

El bloque temático escogido, "La Cuenca Hidrológica", tiene una parte en la que se desarrollan los contenidos conceptuales. La innovación realizada consiste en la introducción de la enseñanza de herramientas, software, bases de datos y servidores on-line y actualizados, que permiten realizar el estudio de la cuenca, además de emplear una nueva metodología docente.

Para ello se ha diseñado un mapa de contenidos y problemas (Figura 3), para la clase "teórica", en el que quedan reflejados contenidos conceptuales, procedimentales y actitudinales, en recuadros con diferentes colores, uno para cada tipo de contenidos. Los contenidos de actitudes no se contemplan en el mapa de contenidos por haberse desarrollado en el tema previo a este de manera específica y con mayor grado de detalle. También se ha establecido una jerarquía en la importancia para cada contenido, representada por el tamaño de mayor a menor según el orden de esta jerarquía.

Se diseñó, asimismo, un mapa de contenidos más específicos para las clases prácticas (Figura 4), enfocadas a modo de "taller conceptual" (Finkel, 2008). 


\section{Mapa de contenidos - Clase de Teoría}

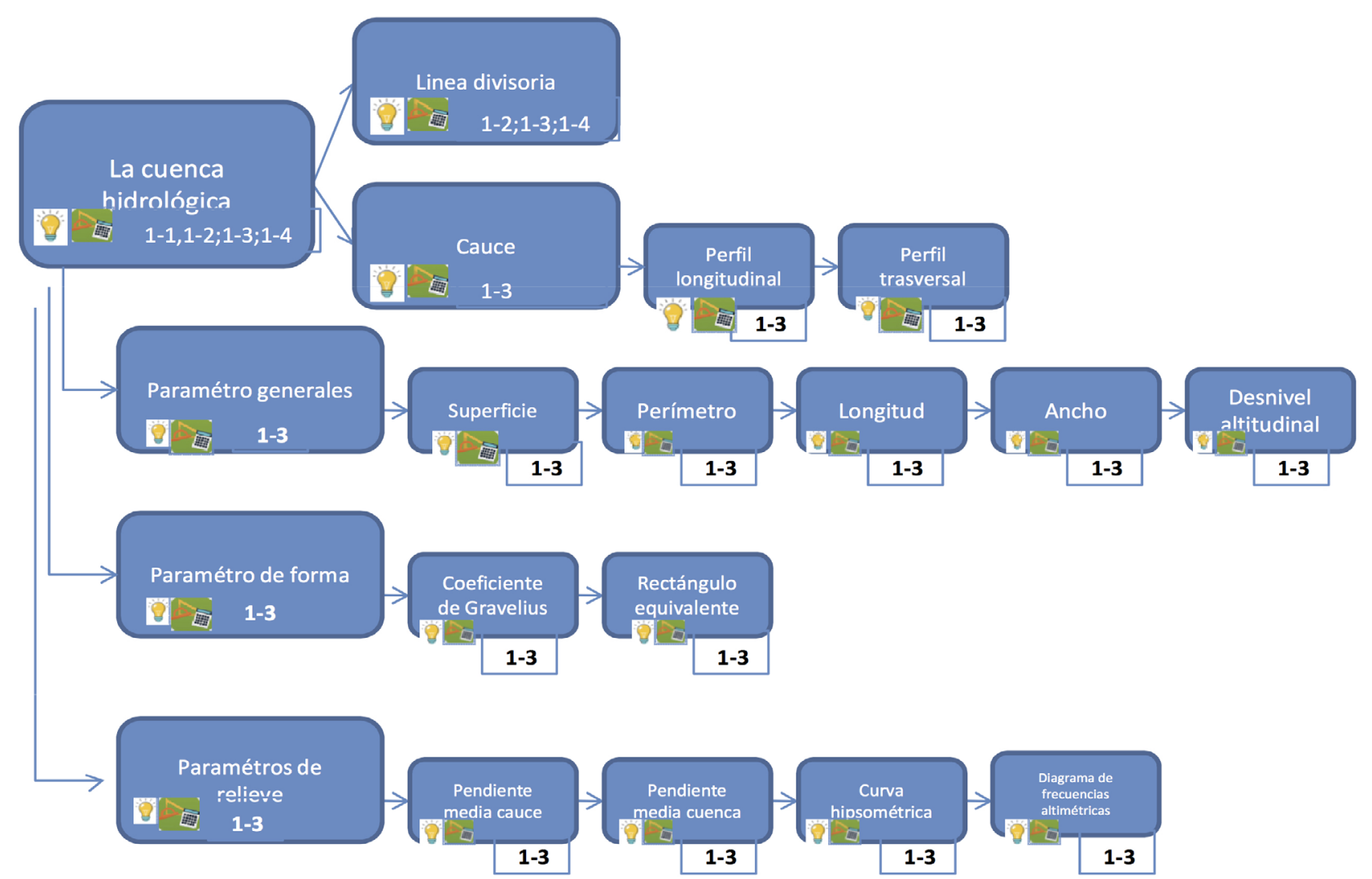

Figura 3. Mapa de contenidos de la clase de teoría.

Jornadas de Formación e Innovación Docente del Profesorado | № 1 (2018)

(C) Esta obra se distribuye con la licencia Creative Commons

cc) (i) $\ominus$ Reconocimiento-NoComercial-SinObraDerivada

Internacional (CC BY-NC-ND 4.0.) 
Paralelamente a la realización del diseño de estos mapas de contenidos se ha diseñado una secuencia temporalizada de actividades para cada una de las sesiones, las cuales están codificadas mediante dos cifras: la primera indica el número de la sesión y la segunda indica el orden en el que va a ser realizada. 
Mapa de contenidos "Talleres conceptuales"

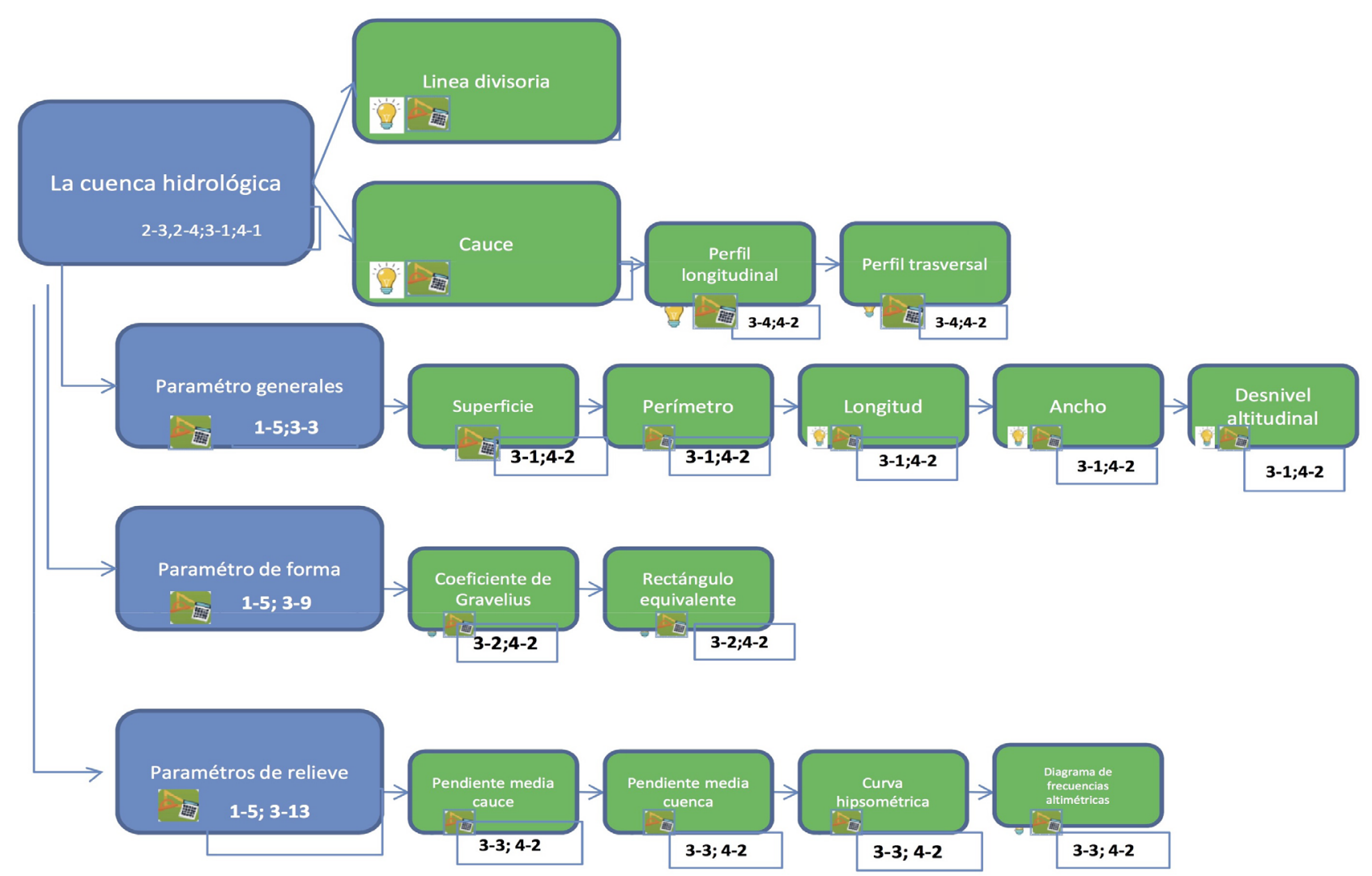

Figura 4. Mapa de contenidos de los "talleres conceptuales".

Jornadas de Formación e Innovación Docente del Profesorado | № 1 (2018)

(C) Esta obra se distribuye con la licencia Creative Commons

cc) (i) $\Theta$ Reconocimiento-NoComercial-SinObraDerivada Internacional (CC BY-NC-ND 4.0.) 
Dentro de los mapas de contenidos, y junto a cada uno de los contenidos que se han representado, se refleja un cuadro con los códigos descritos anteriormente. Con este código podemos buscar en la relación detallada de las actividades presentada más adelante y obtener así más información acerca de la actividad que estamos desarrollando.

La leyenda, junto con los dos mapas de contenidos y actividades diseñados y la relación de las actividades codificadas para alcanzar el desarrollo de estos contenidos se representan en las figuras 3 y 4 , citadas, y en las tablas 1 a 4 se recogen las secuencias de actividades de las 4 sesiones impartidas.

Tabla 1. Secuencia de actividades de la sesión 1 del ciclo de mejora. Clase de "teoría"

\begin{tabular}{|c|l|l|l|}
\hline No & \multicolumn{1}{|c|}{ Descripción } & Temporalización & \multicolumn{1}{c|}{ Contenido } \\
\hline 1.1 & $\begin{array}{l}\text { Reseña de lectura. Texto sobre } \\
\text { la influencia de las avenidas. }\end{array}$ & 15 minutos & Actitudinal \\
\hline 1.2 & $\begin{array}{l}\text { Evaluación de ideas previas. } \\
\text { Cuestionario inicial. }\end{array}$ & 15 minutos & Conceptual \\
\hline 1.3 & $\begin{array}{l}\text { Exposición del tema teórico } \\
\text { “La cuenca hidrológica". }\end{array}$ & 40 minutos & Conceptual \\
\hline 1.4 & $\begin{array}{l}\text { Resolución del problema del } \\
\text { cuestionario inicial. }\end{array}$ & 40 minutos & Conceptual \\
\hline
\end{tabular}

Jornadas de Formación e Innovación Docente del Profesorado | № 1 (2018) Esta obra se distribuye con la licencia Creative Commons Reconocimiento-NoComercial-SinObraDerivada Internacional (CC BY-NC-ND 4.0.) 
Tabla 2. Secuencia de actividades de la sesión 2 del ciclo de mejora. "Taller conceptual".

\begin{tabular}{|c|l|l|l|}
\hline № & \multicolumn{1}{|c|}{ Descripción } & Temporalización & \multicolumn{1}{|c|}{ Contenido } \\
\hline 2.1 & $\begin{array}{l}\text { Presentación de la aplicación } \\
\text { Goolzoom. Interfaz de usuario, } \\
\text { opciones de vista, mapa, satélite. }\end{array}$ & 30 minutos & $\begin{array}{l}\text { Conceptual, } \\
\text { procedimen- } \\
\text { tal }\end{array}$ \\
\hline 2.2 & $\begin{array}{l}\text { Presentación de repositorios de } \\
\text { mapas disponibles. }\end{array}$ & 20 minutos & $\begin{array}{l}\text { Conceptual, } \\
\text { procedimen- } \\
\text { tal }\end{array}$ \\
\hline 2.3 & $\begin{array}{l}\text { Presentación de la cuenca a es- } \\
\text { tudiar. Cuenca del río Verde } \\
\text { (Málaga) }\end{array}$ & 20 minutos & Conceptual \\
\hline 2.4 & $\begin{array}{l}\text { Realización de la práctica on-time } \\
\text { y on-line. }\end{array}$ & 50 minutos & $\begin{array}{l}\text { Conceptual, } \\
\text { procedimen- } \\
\text { tal }\end{array}$ \\
\hline
\end{tabular}

Tabla 3. Secuencia de actividades de la sesión 3 del ciclo de mejora. "Taller conceptual".

\begin{tabular}{|c|l|l|l|}
\hline No & \multicolumn{1}{|c|}{ Descripción } & Temporalización & Contenido \\
\hline 3.1 & $\begin{array}{l}\text { Cálculo utilizando Goolzoom de los } \\
\text { parámetros generales: área, perí- } \\
\text { metro, longitud, ancho, desnivel } \\
\text { altitudinal. }\end{array}$ & $\begin{array}{l}\text { Conceptual, } \\
\text { procedi- } \\
\text { mental }\end{array}$ \\
\hline 3.2 & $\begin{array}{l}\text { Cálculo con Goolzoom de los pa- } \\
\text { rámetros de forma: Coeficiente de } \\
\text { Gravelius y rectángulo equivalente. }\end{array}$ & 20 minutos & $\begin{array}{l}\text { Conceptual, } \\
\text { procedi- } \\
\text { mental }\end{array}$ \\
\hline 3.3 & $\begin{array}{l}\text { Cálculo con Goolzoom de los pará- } \\
\text { metros de relieve: pendiente media } \\
\text { de una cuenca, pendiente media } \\
\text { del cauce, curva hipsométrica e } \\
\text { histograma de frecuencias. }\end{array}$ & $\begin{array}{l}\text { Conceptual, } \\
\text { procedi- } \\
\text { mental }\end{array}$ \\
\hline 3.4 & $\begin{array}{l}\text { Cálculo con Goolzoom de los pará- } \\
\text { metros de la red de drenaje: perfil } \\
\text { longitudinal y sección transversal, } \\
\text { densidad de drenaje. }\end{array}$ & 20 minutos & $\begin{array}{l}\text { Conceptual, } \\
\text { procedi- } \\
\text { mental }\end{array}$ \\
\hline
\end{tabular}

Jornadas de Formación e Innovación Docente del Profesorado | № 1 (2018) Esta obra se distribuye con la licencia Creative Commons Reconocimiento-NoComercial-SinObraDerivada Internacional (CC BY-NC-ND 4.0.) 
Tabla 4. Secuencia de actividades de la sesión 4 del ciclo de mejora. Evaluación final.

\begin{tabular}{|c|l|l|l|}
\hline No & \multicolumn{1}{|c|}{ Descripción } & Temporalización & \multicolumn{1}{c|}{ Contenido } \\
\hline 4.1 & Entrega de la práctica. & 5 minutos & $\begin{array}{l}\text { Conceptual, } \\
\text { procedimental }\end{array}$ \\
\hline 4.2 & $\begin{array}{l}\text { Realización en clase de la prác- } \\
\text { tica en la que se realiza un es- } \\
\text { tudio completo de la cuenca, } \\
\text { calculando todos los parámetros } \\
\text { vistos en las sesiones anteriores. } \\
\text { Entrega on-line. }\end{array}$ & $\begin{array}{l}\text { Conceptual, } \\
\text { procedimental }\end{array}$ \\
\hline
\end{tabular}

\section{Aplicación del Ciclo de Mejora Docente}

El Ciclo de Mejora Docente se ha desarrollado en 4 sesiones, como se ha descrito en apartados anteriores. La introducción de esta nueva metodología de trabajo, con clases y actividades pensadas y programadas, haciendo que los alumnos "sean protagonistas" en primera persona de su aprendizaje, interactuando con el profesor y trabajando "on-line" y "on-time", ha creado un clima de trabajo muy enriquecedor en el que se notaba una satisfacción con el trabajo desarrollado, con la forma de aprender y trabajar que más tarde han expresado los alumnos. Se han seguido las indicaciones dadas por Ken Bain (2007) en Lo que hacen los mejores profesores de universidad y se han tomado ideas del material Elaboración de material docente para la mejora del aprendizaje y adquisición de habilidades en Hidráulica e Hidrología (Pardo, 2016). Las actividades se han realizado casi de acuerdo con la programación prevista, aunque los tiempos inicialmente programados no se han cumplido, y no se han encontrado apenas dificultades en el desarrollo de estas actividades.

Para evaluar el aprendizaje de los alumnos se han comparado sus conocimientos iniciales y finales mediante dos cuestionarios (Rivero y Porlán, 2017).

Jornadas de Formación e Innovación Docente del Profesorado I № 1 (2018) Esta obra se distribuye con la licencia Creative Commons 
En el cuestionario inicial los contenidos conceptuales evaluados son: Concepto de cuenca hidrológica, delimitación, trazado de divisorias y variables que influyen en la generación de caudal. En cuanto a los contenidos procedimentales, no se procede a su evaluación mediante cuestionario debido a que el primer día se preguntó verbalmente si habían trabajado con herramientas informáticas que permitan realizar el estudio de las cuencas como Goolzoom y ArcMap, contestando por unanimidad que no y que las desconocían por completo la totalidad de los alumnos. Tomando como base la figura 5 se plantea el cuestionario inicial, con las siguientes cuestiones:

1. Define el concepto de cuenca hidrológica.

2. Una vez señalado por el profesor, identificar el Río verde y dibujar a mano alzada lo que consideras que es su "Cuenca Hidrológica en el punto de desembocadura en el mar". Igualmente el profesor te señalará otro río, del cual debes dibujar a mano alzada su cuenca hidrológica en el punto que te indique.

3. ¿Qué variables crees que influyen en el Caudal que llega al punto de la desembocadura en el mar?

HIDROLOGÍA:Evaluación previa

En la figura representada se puede observar las capas de rios y de curvas de nivel de una determinada zona del litoral

mediterráneo.

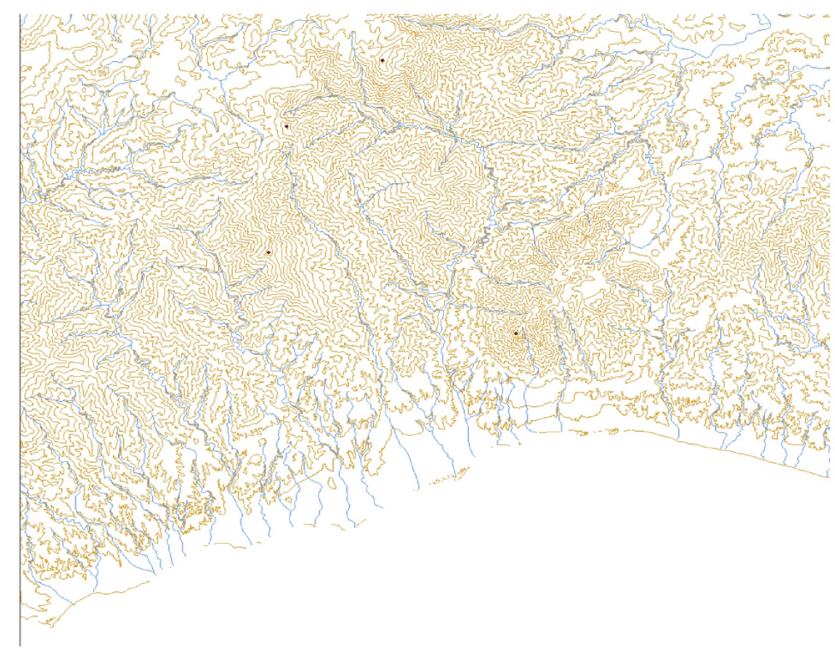

Figura 5. Imagen para el cuestionario inicial.

Jornadas de Formación e Innovación Docente del Profesorado | № 1 (2018) Esta obra se distribuye con la licencia Creative Commons 
Para evaluar el nivel de conocimiento de los alumnos en las respuestas a cada pregunta, se establecen 3 niveles: inicial, medio y final. El cuestionario fue realizado por 4 alumnos mostrándose a continuación en figura la representación gráfica de los resultados obtenidos para cada una de las tres preguntas.

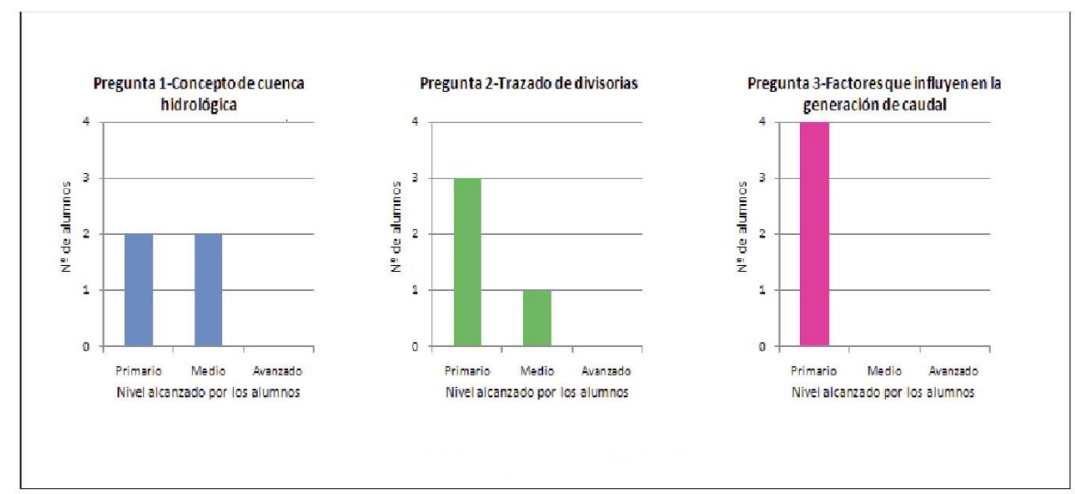

Figura 6. Representación gráfica de los resultados del cuestionario inicial.

La evaluación de los conocimientos al final del Ciclo de Mejora se ha realizado mediante un cuestionario-práctica final en el que, con las herramientas informáticas vistas en las sesiones anteriores, los alumnos deben realizar su propio estudio de la Cuenca Hidrológica. Se utilizan los ordenadores en el Centro de Cálculo con las herramientas y software necesario. Los conceptos que se evalúan son: utilización de la herramienta Goolzoom para crear mapas de cuenca; concepto de cuenca hidrológica - trazado de divisorias; perfiles longitudinales y trasversales de un río, parámetros generales y parámetros de forma. El cuestionario entregado se muestra en la figura 7. 


\section{HIDROLOGÍA:Evaluación final}

En la figura representada se puede observar las capas de rios y de curvas de nivel de una determinada zona del litoral mediterráneo en la que se encuentra el Río Verde y su correndiente cuenca hidrológica.

Se pide, utilizando el software informático Goolzoom

1- Utilización de la herramienta Goolzoom diversos mapas y vistas de la zona.

2- Dibuja la divisoria de la cuenca del Río Verde.

3- Traza y calcula el perfil longitunidal y tres perfiles trasversales del río verde en varios trazmos.

4- Calcula los parámetros generales de la cuenca.

5- Calcula sus parámetros de forma de la cuenca.

6- Calcula los parámetros de relieve de la cuenca.

Figura 7. Cuestionario final.

Para valorar las respuestas se establecen igualmente 3 niveles de conocimiento para cada pregunta: inicial, medio y avanzado. La práctica fue realizada por 4 alumnos. En la figura 8, se representan gráficamente a continuación.
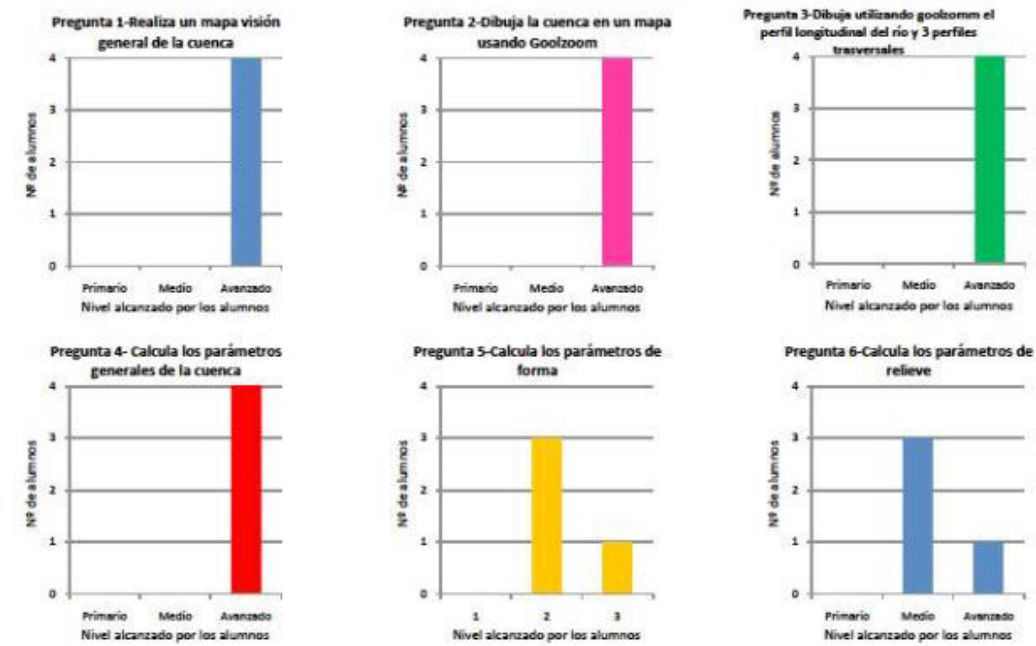

Figura 8. Representación gráfica de los resultados del cuestionario final.

Jornadas de Formación e Innovación Docente del Profesorado | № 1 (2018)

cC (i) Esta obra se distribuye con la licencia Creative Commons Reconocimiento-NoComercial-SinObraDerivada 
Como puede observarse los resultados son muy satisfactorios, pues la totalidad de los alumnos llegan al nivel avanzado en 4 de las 6 cuestiones analizadas en el cuestionario final, alcanzando un nivel medio 3 de los 4 alumnos en las otras 2 cuestiones y una nivel avanzado 1 alumno en estas dos últimas cuestiones, por lo que se puede concluir que los resultados son bastante satisfactorios.

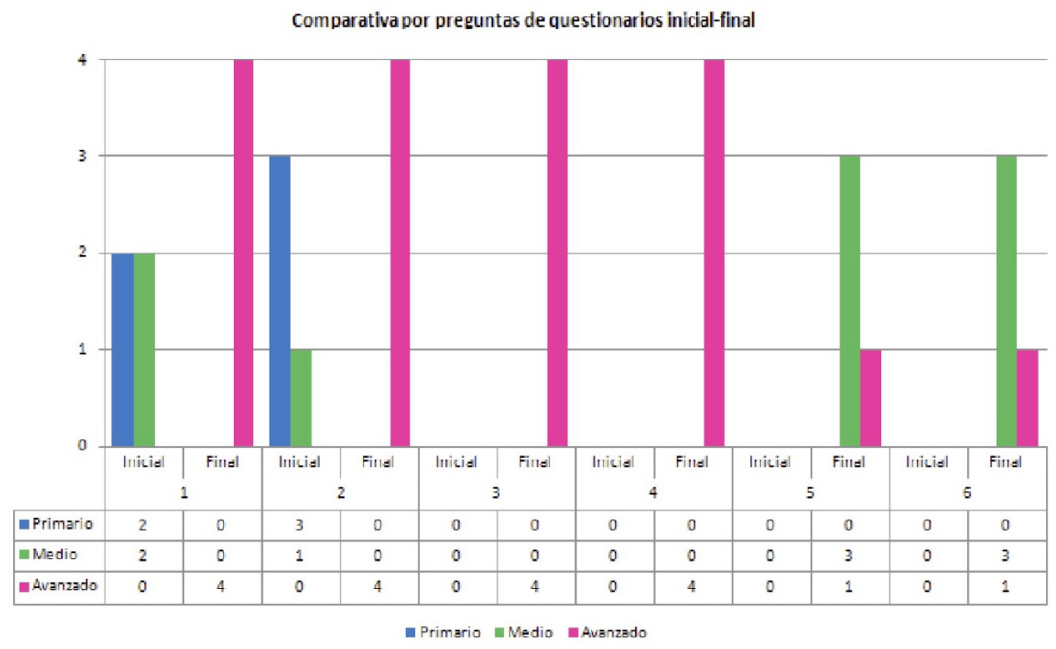

Figura 9. Comparación de los resultados de los cuestionarios inicial y final.

Aunque los cuestionarios inicial y final no coinciden existe un paralelismo entre algunas preguntas que, de manera diferente, evalúan los mismos conceptos, lo que nos permite comparar la evolución del aprendizaje en los contenidos conceptuales. La evaluación de los contenidos procedimentales se muestra en los gráficos de la figura 8 , sabiendo que el nivel inicial de todos los alumnos era nulo. En la figura 9, en la que se comparan los resultados de los cuestionarios inicial y final, se representa la evolución del aprendizaje por preguntas. 


\section{Evaluación del Ciclo de Mejora Docente}

El empleo de talleres conceptuales y cambiar el modelo de enseñanza "dando clase con la boca cerrada" (Finkel, 2008) me parece muy acertado, y los resultados obtenidos resultan exitosos tanto para alcanzar los objetivos de contenidos conceptuales, procedimentales y actitudinales como también por la manera en que se alcanzan estos objetivos, al ser la forma del aprendizaje mucho más amena, divertida y por tanto enriquecedora y muy positiva para los alumnos y reconfortante para el profesor, en definitiva, más didáctica.

La forma de trabajar los contenidos con mapas de contenidos y problemas es muy efectiva para realizar posteriormente la programación de las actividades y la evaluación final de los alumnos. Esta metodología de trabajo hace que tengamos un "mapa" como guía de lo que queremos enseñar de una forma más clara, ordenada y estructurada, lo que indudablemente repercute en una mejor práctica docente.

La programación y temporización de las actividades, el buscarle un sentido a cada una de ellas, el realizar preguntas que despierten y mantengan la atención durante las clases, suponen un gran ejercicio de reflexión y preparación por parte del docente, que se ve altamente recompensado con la satisfacción de saber que los alumnos aprenden al hacerles pensar y trabajar y con un altísimo nivel de satisfacción por parte de ellos y consecuentemente del profesor.

El tratamiento de los datos y la representación de los resultados obtenidos mediante gráficos incita a la reflexión posterior sobre diversas variables, como pueden ser: el método empleado en el aprendizaje, el sistema de enseñanza, los objetivos alcanzados, etc. La elaboración de escaleras y gráficos comparativos es interesante para analizar nuestra práctica docente y continuar con un proceso de mejora continua.

Jornadas de Formación e Innovación Docente del Profesorado | № 1 (2018) Esta obra se distribuye con la licencia Creative Commons 
En definitiva, la aplicación del Ciclo de Mejora Docente supone un punto de inflexión en mi manera de concebir la docencia aportando muchas ideas que abarcan desde la disposición de las aulas, la preparación de las clases, los talleres conceptuales, la forma de interactuar con los alumnos, de trabajar, de evaluar, de "dar clase con la boca cerrada" que más que un fin me parecen un principio para empezar a cambiar y seguir preparando otros ciclos de Mejora.

En esa línea, el programa de formación docente me parece muy acertado y útil en su conjunto pues ofrece la posibilidad real de aprender a "hacer docencia" y de mejorar en nuestra profesión docente siendo una guía para afrontar mejor la práctica docente desde un modelo innovador de enseñanza. La realización del Ciclo de Mejora supone un inicio en el que se han puesto bases sólidas para empezar a andar un nuevo camino de mejora continua. De cara al futuro, la REFID supone un entorno seguro para realizar esta mejora continua de la práctica docente empleando nuevas técnicas innovadoras, recibiendo apoyo y guías de trabajo de otros compañeros, más experimentados o no, pero que de una forma u otra permite compartir experiencias que enriquecen mucho y hacen que crezcamos todos como profesionales.

Jornadas de Formación e Innovación Docente del Profesorado | № 1 (2018) Esta obra se distribuye con la licencia Creative Commons 


\section{Referencias bibliográficas}

Bain, K. (2007). Lo que hacen los mejores profesores de universidad. Valencia: Universidad de Valencia.

De Alba, N. y Porlán, R. (2017). La metodología de enseñanza. En R. Porlán (coord.), Enseñanza universitaria. Cómo mejorarla (pp. 37-54). Madrid: Morata.

Finkel, D. (2008). Dar clase con la boca cerrada. Valencia: Universidad de Valencia.

Pardo, M. A. (2016). Elaboración de material docente para la mejora del aprendizaje y adquisición de habilidades en Hidráulica e Hidrología. Universidad de Alicante. Instituto de Ciencias de la Educación.

Rivero, A. y Porlán, R. (2017). La evaluación en la enseñanza universitaria. En R. Porlán (coord.), Enseñanza Universitaria. Cómo mejorarla (pp. 77-91). Madrid: Morata.

Jornadas de Formación e Innovación Docente del Profesorado | № 1 (2018) Esta obra se distribuye con la licencia Creative Commons 